\title{
Effect of thyroidectomy in patients with tracheal compression
}

\author{
Sinan Koycegiz ${ }^{1}$ (D), Vahit Mutlu² (1), Fatma Atalay ${ }^{3}$ (1), \\ Ayhan Kars ${ }^{3 *}$ (1) Kubra Topal $^{3}$ (D), Mustafa Yesilyurt ${ }^{4}$ (1)
}

\section{SUMMARY}

OBJECTIVE: Various therapeutic methods are employed to reduce thyroid gland compression of the trachea. Differences in the amount of shrinkage in the thyroid gland, in the amount of amelioration of tracheal compression, and in the amount of fibrosis after treatment may occur with these different methods. Although the compression of the trachea decreases after thyroidectomy, the number of studies showing the extent of this is limited. The purpose of this study was to investigate the effect of thyroidectomy performed due to tracheal compression, to reveal the extent of improvement using magnetic resonance imaging (MRI), and to evaluate our results.

METHODS: In total, 30 patients, i.e., 24 women and 6 men, with tracheal compression secondary to thyroid gland enlargement and undergoing total thyroidectomy were included in this study. MRI performed before surgery and 6 months after surgery. The amount of deviation from the tracheal midline and the tracheal lateral and anteroposterior (AP) diameters were measured, compared, and subjected to statistical analysis.

RESULTS: Statistical analysis revealed significant differences between pre- and postoperative tracheal deviations, and lateral and AP diameters $(p<0.001, p<0.001$, and $p=0.006$, respectively). Histopathologically, benign or malignant pathology caused no significant difference in the postoperative improvement of tracheal anatomy ( $p=0.348$ and $p=0.148$, respectively).

CONCLUSIONS: Thyroidectomy performed due to tracheal compression provides significant improvement in tracheal anatomy. Due to its rapid and effective results, thyroidectomy should be one of the first options considered in the treatment of thyroid diseases with compression findings.

KEYWORDS: Goiter. Thyroidectomy. Tracheal stenosis.

\section{INTRODUCTION}

The term "goiter" refers to the abnormal growth of the thyroid gland. Growth of the thyroid gland can lead to symptoms such as swelling in the neck, shortness of breath, hoarseness, and swallowing difficulty by compressing surrounding structures such as the trachea, esophagus, recurrent laryngeal nerve, and the internal jugular vein. Due to slow progression and patient adaptation, it may often not be diagnosed. Objective findings in the presence of tracheal compression emerge in respiratory function tests when approximately $50 \%$ of the trachea is affected ${ }^{1}$.

Tests used to evaluate the volume of an enlarged thyroid gland include ultrasonography, computed tomography, magnetic resonance imaging (MRI), and thyroid scintigraphy. Due to its high sensitivity and the greater anatomical detail it

\footnotetext{
${ }^{1}$ Maresal Cakmak State Hospital, Head and Neck Surgery, Department of Otorhinolaryngology - Erzurum, Turkey.

${ }^{2}$ Ataturk University Faculty of Medicine, Head and Neck Surgery, Department of Otorhinolaryngology - Erzurum, Turkey.

${ }^{3}$ Kastamonu University Faculty of Medicine, Head and Neck Surgery, Department of Otorhinolaryngology - Kastamonu, Turkey.

${ }^{4}$ Patnos State Hospital, Department of Radiology - Agri, Turkey.

*Corresponding author: drakars25@hotmail.com

Conflicts of interest: the authors declare there is no conflicts of interest. Funding: none.

Received on July 26, 2021. Accepted on August 18, 2021.
} 
provides, MRI is regarded as more useful in treatment planning and follow-up compared with other methods ${ }^{2,3}$.

Total thyroidectomy and radioactive iodine therapy are the therapeutic methods that can be applied in patients with goiter and tracheal compression ${ }^{4,5}$. Differences may be observed in the extent of shrinkage in the thyroid gland, the amount of tracheal compression amelioration, and the amount of fibrosis after treatment among the different therapeutic methods employed in reducing thyroid gland compression of the trachea. Several studies have shown that thyroidectomy contributes to tracheal airflow ${ }^{6,7}$. However, the number of studies concerning changes occurring in the structure of the trachea following thyroidectomy is insufficient.

The purpose of this study was to investigate the effect of thyroidectomy performed due to tracheal compression, reveal the extent of improvement using MRI, and evaluate our results.

\section{METHODS}

The study commenced following the receipt of Ataturk University Medical Faculty Clinical Research Ethical Committee approval (decision no. 15, session no. 07, dated November 29, 2018). A total of 30 patients with tracheal compression secondary to thyroid gland enlargement and who had undergone total thyroidectomy at the Ataturk University Medical Faculty Ear, Nose, and Throat Department, Turkey, were included in this study. Patients with previous histories of neck surgery, revision cases, who were undergoing lobectomy and hemithyroidectomy, with a history of neck radiotherapy, or receiving radioactive iodine therapy were excluded.

Extracapsular total thyroidectomy was performed on all patients using the same standardized technique. Age, gender, preoperative and postoperative deviation in the trachea and compression regions, and histopathological results were recorded for all the cases included in this study.

MRI of the neck (Siemens Magnetom Avanto 1.5 T MRI Scanner) was performed in the axial, sagittal, and coronal planes from the epiglottis to the carina. This procedure was performed in a mean 30 days before surgery and 180 days postoperatively. T1- and T2-weighted images were obtained with 1.5 T MRI at a section thickness of $5 \mathrm{~mm}$. Coronal plane T1or T2-weighted MRI was used to measure the degree of deviation of the trachea. Before measurement, following the selection of the section in which the trachea was seen most clearly and in its entirety, the air intensity was enhanced by inclusion in the minimum intensity projection (MinIP) algorithm, and measurements were subsequently performed. At preoperative MRI, the midline of the neck was identified, and the area where the trachea deviated most from the midline was marked.
The distance between the trachea at this level and the midline was then measured manually.

At postoperative MRI, the amount of deviation improvement was determined by measuring the distance between the trachea at the same level and the midline. The amount of tracheal compression and the degree of postoperative improvement were determined using T1- or T2-weighted MRI in the axial plane. At preoperative MRI, the narrowest part of the trachea in the regions where the thyroid gland compressed the trachea was identified, and the lateral and anteroposterior (AP) tracheal diameters were measured manually. At postoperative MRI, the lateral and AP diameters were measured at the same level to determine the amount of improvement in the tracheal lumen.

The study data were recorded and analyzed using SPSS version 22.0 software. Descriptive statistics were shown as mean \pm standard deviation, median (minimum-maximum), percentage, and frequency distributions. The normality of the distribution of variables was tested using the histogram chart method based on the Kolmogorov-Smirnov test and skewness coefficients. The Wilcoxon test, Mann-Whitney $U$ test, and Spearman's Rho correlation test were employed to evaluate variables before and after surgery. The $\mathrm{p}<0.05$ were regarded as statistically significant.

\section{RESULTS}

A total of 30 patients were included in this study, i.e., 24 $(80 \%)$ women and $6(20 \%)$ men. The patients' mean age was $50.23 \pm 10.82$ years, ranging between 29 and 70 . Based on the histopathology results, $53.3 \%$ of the removed thyroid tissues were malignant, and $46.7 \%$ were benign. The mean specimen weight of the 30 patients was $113.27 \pm 54.50 \mathrm{~g}$, ranging between 50 and $262 \mathrm{~g}$.

MRI images of changes in preoperative and postoperative tracheal deviations are shown in Figure 1A, and those of changes in preoperative and postoperative lateral and $\mathrm{AP}$ tracheal diameters are shown in Figure 1B. AP and lateral tracheal diameters, levels of deviation, and amounts of improvement measured at pre- and postoperative MRI are summarized in Table 1. Statistically significant differences were observed between preand postoperative tracheal deviations, tracheal lateral diameters, and tracheal AP diameters $(\mathrm{p}<0.001, \mathrm{p}<0.001$, and $\mathrm{p}=0.006$, respectively) (Table 2).

Benign or malignant thyroid diseases had no significant effect on the levels of deviation and tracheal AP diameter, although lateral tracheal diameters were significantly lower in benign masses ( $\mathrm{p}=0.190, \mathrm{p}=0.771$, and $\mathrm{p}=0.011$, respectively). Benign or malignant thyroid diseases produced no significant 
difference in terms of postoperative improvement of tracheal deviation, lateral tracheal diameter, or AP tracheal diameter ( $\mathrm{p}=0.348, \mathrm{p}=0.148$, and $\mathrm{p}=0.950$, respectively).

The substernal extension of the thyroid gland was present in 18 cases $(60 \%)$. The postoperative improvement of tracheal

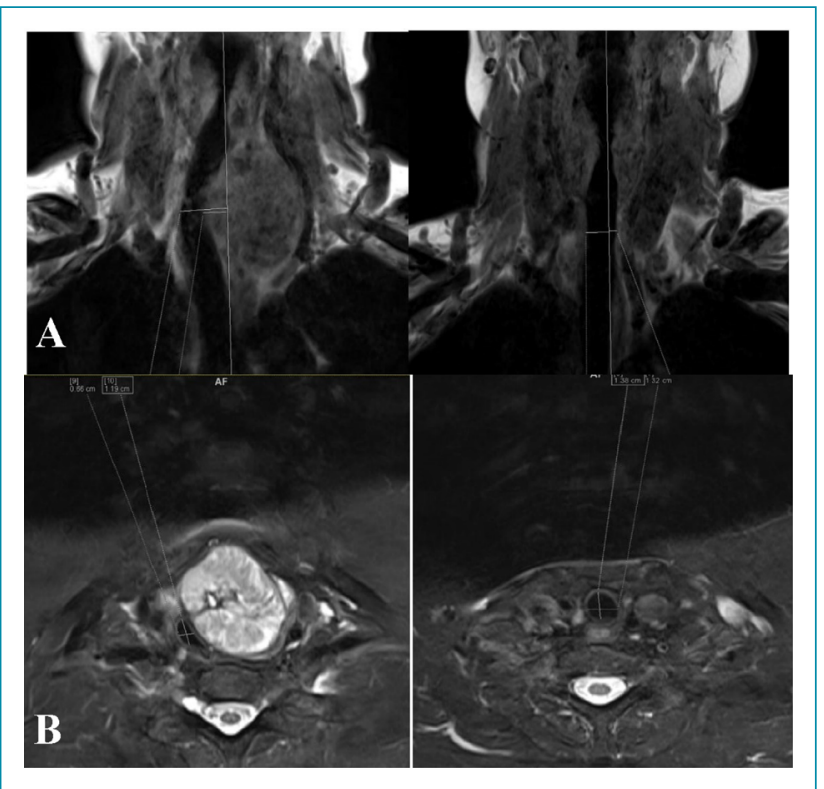

Figure 1. (A) Magnetic resonance image of the change in pre- and postoperative tracheal deviations. (B) Magnetic resonance image of the change in pre- and postoperative tracheal lateral and anteroposterior diameters. deviation was significantly greater in patients with the substernal extension of the thyroid gland $(\mathrm{p}=0.034)$. No significant difference was observed in the amount of improvement in lateral or AP tracheal diameters between cervical and substernal goiters ( $\mathrm{p}=0.241$ and $\mathrm{p}=0.226$, respectively).

No significant relationship was observed between age and degrees of improvement in tracheal deviation or lateral tracheal diameter. However, a significant moderate correlation was observed between age and the amount of improvement in AP diameter. Improvement in AP diameter increased with age $(\mathrm{p}=0.121, \mathrm{p}=0.608$, and $\mathrm{p}=0.025$, respectively).

The weight of tracheal tissue that was removed had no significant effect on the amount of improvement in tracheal deviation or lateral and AP diameters $(\mathrm{p}=0.545, \mathrm{p}=0.080$, and $\mathrm{p}=0.211$, respectively). Histopathological findings did not significantly affect the size of thyroid tissue removed during surgery as a result of pathology $(\mathrm{p}=0.371)$.

\section{DISCUSSION}

Thyroidectomy is the most frequently employed therapeutic technique in goiter patients with compression symptoms. One potential alternative to surgery is radioactive iodine therapy 5 . However, radioactive iodine therapy involves undesirable radiation-associated side-effects, such as thyroiditis, transient hyperthyroidism, Graves-like hyperthyroidism, and sometimes a $15-25 \%$ increase in thyroid dimensions. In addition,

Table 1. Anteroposterior and lateral tracheal diameters, deviation, and improvements therein measured using preoperative and postoperative magnetic resonance imaging (minimum, maximum, and meantstandard deviation).

\begin{tabular}{l|c|c|c} 
& Minimum & Maximum & Mean \pm standard deviation \\
\hline Preoperative deviation $(\mathrm{mm})$ & 7.0 & 28.0 & $13.96 \pm 4.61$ \\
\hline Preoperative lateral diameter $(\mathrm{mm})$ & 6.0 & 15.0 & $10.97 \pm 2.42$ \\
\hline Preoperative AP diameter $(\mathrm{mm})$ & 6.3 & 30.0 & $13.26 \pm 4.22$ \\
\hline Postoperative deviation $(\mathrm{mm})$ & 2.5 & 13.5 & $7.93 \pm 2.86$ \\
\hline Postoperative lateral diameter $(\mathrm{mm})$ & 12.0 & 18.0 & $14.87 \pm 1.65$ \\
\hline Postoperative AP diameter $(\mathrm{mm})$ & 11.0 & 21.0 & $14.29 \pm 2.48$ \\
\hline Deviation improvement $(\mathrm{mm})$ & 0.50 & 19.00 & $6.03 \pm 4.34$ \\
\hline Lateral diameter improvement $(\mathrm{mm})$ & 0.00 & 10.00 & $3.90 \pm 2.48$ \\
\hline AP diameter improvement $(\mathrm{mm})$ & -9.00 & 7.00 & $1.03 \pm 2.78$ \\
\hline
\end{tabular}

Table 2. Preoperative and postoperative tracheal deviations, as well as lateral and anteroposterior tracheal diameters.

\begin{tabular}{l|c|c|c} 
& Preoperative & Postoperative & p-value \\
\hline Tracheal deviation measure $(\mathrm{mm})$ & $13.25(7.00-28.00)$ & $7.75(2.5-13.5)$ & $<0.001$ \\
\hline Tracheal lateral diameter $(\mathrm{mm})$ & $11.00(6.00-15.00)$ & $15.00(12.00-18.00)$ & $<0.001$ \\
\hline Tracheal AP diameter $(\mathrm{mm})$ & $12.50(6.30-30.00)$ & $14.00(11.00-21.00)$ & 0.006 \\
\hline
\end{tabular}

AP: anteroposterior. 
a decrease in thyroid volume occurs over the long term. The absence of such side-effects and immediate resolution of compression symptoms are the important advantages of goiter patients undergoing thyroidectomy ${ }^{8}$. Significant improvements in tracheal stenosis and deviation were also observed following thyroidectomy performed due to tracheal compression in this study.

The internal diameters of the trachea are of vital importance in the passage of air to the lungs via the upper respiratory tracts. According to the Hagen-Poiseuille law, the resistance to flow through a tube is inversely proportional to the fourth power of the radius of the tube if the flow is laminar. At higher rates, the flow can become turbulent and further increase resistance. Deformities arising in the trachea following compression produce a significant increase in airflow resistance by reducing the space of the tracheal airway. A $50 \%$ decrease in the internal diameter increases resistance 16-fold and up to 32 times if a turbulent flow develops. Following thyroidectomy, the deformity in the trachea is improved, and the airflow decreases significantly?.

The changes in tracheal parameters and respiratory functions which follow other therapeutic methods employed to reduce the effect of thyroid gland pressure have also been investigated. Improvements have been shown in tracheal parameters and respiratory functions 6-12 months after treatment in patients receiving radioactive iodine therapy, either alone or in combination with recombinant human thyroid-stimulating hormone (TSH) $(\mathrm{rhTSH})^{5,10,11}$. However, the number of studies on the extent of postoperative improvement in areas of tracheal stenosis and tracheal deviation causing preoperative symptoms following thyroidectomy performed due to tracheal compression is limited. Previous studies have evaluated the effect of thyroidectomy on upper airway obstruction using pulmonary function tests ${ }^{6,7}$.

Sorensen et al. showed that thyroidectomy produced significant improvements in tracheal anatomy and airflow in patients with benign nodular goiter and tracheal compression and that this was also correlated with an improved quality of life. Those authors also reported that the improvement in tracheal parameters was greater in patients with substernal goiter ${ }^{6}$. Similarly in this study, greater improvement was observed in patients with substernal goiter. Additionally, those authors reported a marked relationship between benign thyroid tissue volume and improvement in both tracheal anatomy and also airflow ${ }^{6}$. The amount in line with the weight of the thyroid tissue removed in this study, although no significant relationship was determined between the weight of tissue removed and improvement in tracheal deviation or stenosis.

Greenblatt et al. evaluated swallowing functions in goiter patients after thyroidectomy using the swallowing quality of life questionnaire and reported significant improvement in swallowing functions in the postoperative period ${ }^{12}$. Wang et al. showed that all deviated tracheas returned to their normal positions after $2-3$ months postoperatively and that mean tracheal diameters increased significantly on the follow-up radiographs of goiter patients with retrosternal extension ${ }^{13}$.

Ayabe et al. reported that performing hemithyroidectomy due to acute tracheal obstruction resulting from a large benign goiter in an 80-year-old woman and that the tracheal lumen returned to normal postoperatively although malacia was present in the tracheal wall due to prolonged compres$\operatorname{sion}^{14}$. Geelhoed reported the subsequent course and outcome in patients undergoing thyroidectomy due to tracheal compression and subsequently developing tracheomalacia ${ }^{15}$. Tracheomalacia is a troubling problem that makes thyroidectomy difficult in patients with longstanding or recurrent tracheal compression. No tracheomalacia or associated problem was observed in any patients in this study.

One particular strength of this study is that both benign and malignant diseases were included. The principal limitations are the low patient number, female predominance in terms of gender distribution, the fact that no analysis regarding this could be performed, and the single-center nature of the research. This study should currently be supported by further multicenter studies with larger case numbers.

\section{CONCLUSIONS}

Thyroidectomy due to tracheal compression was found to provide significant improvements in tracheal deviation, compression, and deformity. We think that, due to its rapid and effective results, thyroidectomy should represent the first treatment option in thyroid diseases that compress the surrounding tissues and that urgent thyroidectomy will enhance patient comfort and quality of life.

\section{ACKNOWLEDGMENTS}

The authors thank Mr. Carl Austin Nino Rossini for his precious contribution.

\section{AUTHORS" CONTRIBUTIONS}

SK: Conceptualization, Data curation, Formal analysis, Investigation, Methodology, Project administration, Resources, Software, Supervision, Validation, Visualization, Writing - original draft, Writing - review \& editing. VM: Conceptualization, Data curation, Formal analysis, Investigation, Methodology, Project administration, Resources, Software, Supervision, 
Validation, Visualization, Writing - original draft, Writing - review \& editing. FA: Data curation, Formal analysis, Methodology, Resources, Validation, Visualization, Writing original draft, Writing - review \& editing. AK: Conceptualization, Data curation, Formal analysis, Methodology, Resources, Software, Validation, Visualization, Writing - original draft,
Writing - review \& editing. KT: Data curation, Formal analysis, Methodology, Software, Validation, Visualization, Writing original draft, Writing - review \& editing. MY: Data curation, Investigation, Methodology, Project administration, Software, Validation, Visualization, Writing - original draft, Writing review \& editing.

\section{REFERENCES}

1. Menon SK, Jagtap VS, Sarathi V, Lila AR, Bandgar TR, Menon $P S$, et al. Prevalence of upper airway obstruction in patients with apparently asymptomatic euthyroid multi nodular goitre. Indian J Endocrinol Metab. 2011;15(Suppl 2):S127-31. https:// doi.org/10.4103/2230-8210.83351

2. Belardinelli L, Gualdi G, Ceroni L, Guadalaxara A, Polettini E, Pappalardo G. Comparison between computed tomography and magnetic resonance data and pathologic findings in substernal goiters. Int Surg. 1995;80(1):65-9. PMID: 7657496

3. Huysmans DA, Haas MM, van den Broek WJ, Hermus AR, Barentsz JO, Corstens FH, et al. Magnetic resonance imaging for volume estimation of large multinodular goitres: a comparison with scintigraphy. Br J Radiol. 1994;67(798):519-23. https:// doi.org/10.1259/0007-1285-67-798-519

4. Efremidou El, Papageorgiou MS, Liratzopoulos N, Manolas KJ. The efficacy and safety of total thyroidectomy in the management of benign thyroid disease: a review of 932 cases. Can J Surg. 2009;52(1):39-44. PMID: 19234650

5. Huysmans DA, Hermus AR, Corstens FH, Barentsz JO, Kloppenborg PW. Large, compressive goiters treated with radioiodine. Ann Intern Med. 1994;121(10):757-62. https:// doi.org/10.7326/0003-4819-121-10-199411150-00005

6. Sorensen JR, Lauridsen JF, Døssing H, Nguyen N, Hegedüs L, Bonnema SJ, et al. Thyroidectomy improves tracheal anatomy and airflow in patients with nodular goiter: a prospective cohort study. Eur Thyroid J. 2017;6(6):307-14. https://doi. org/10.1159/000480348

7. Sørensen JR, Hegedüs L, Kruse-Andersen S, Godballe C, Bonnema SJ. The impact of goitre and its treatment on the trachea, airflow, oesophagus and swallowing function. A systemic review. Best Pract Res Clin Endocrinol Metab. 2014;28(4):481-94. https:// doi.org/10.1016/j.beem.2014.03.002

8. Fast S, Nielsen VE, Bonnema SJ, Hegedüs L. Time to reconsider nonsurgical therapy of benign non-toxic multinodular goitre: focus on recombinant human TSH augmented radioiodine therapy. Eur J Endocrinol. 2009;160(4):517-28. https://doi. org/10.1530/EJE-08-0779

9. Hartnick CJ, Cotton RT. Pediatric otolaryngology. In: Bluestone $C D$, Stool SE, eds. Stridor and airway obstruction. $4^{\text {th }}$ ed. Philadelphia: WB Saunders; 2002. p. 1437-47.

10. Bonnema SJ, Nielsen VE, Boel-Jørgensen H, Grupe $P$, Andersen $\mathrm{PB}$, Bastholt $\mathrm{L}$, et al. Improvement of goiter volume reduction after $0.3 \mathrm{mg}$ recombinant human thyrotropin-stimulated radioiodine therapy in patients with a very large goiter: a double-blinded, randomized trial. J Clin Endocrinol Metab. 2007;92(9):3424-8. https://doi.org/10.1210/jc.2007-0501

11. Graf $H$, Fast $S$, Pacini F, Pinchera $A$, Leung $A$, Vaisman $M$, et al. Modified-release recombinant human TSH (MRrhTSH) augments the effect of (131) I therapy in benign multinodular goiter: results from a multicenter international, randomized, placebocontrolled study. J Clin Endocrinol Metab. 2011;96(5):1368-76. https://doi.org/10.1210/jc.2010-1193

12. Greenblatt DY, Sippel R, Leverson G, Frydman J, Schaefer S, $\mathrm{Chen} \mathrm{H}$. Thyroid resection improves perception of swallowing function in patients with thyroid disease. World J Surg. 2009;33(2):255-60. https://doi.org/10.1007/s00268-0089837-9

13. Wang LS, Shai SE, Fahn HJ, Chan KH, Chen MS, Huang MS. Surgical management of substernal goiter. Scand J Thorac Cardiovasc Surg. 1994;28(2):79-83. https://doi. org/10.3109/14017439409100167

14. Ayabe H, Kawahara K, Tagawa Y, Tomita M. Upper airway obstruction from a benign goiter. Surg Today. 1992;22(1):8890. https://doi.org/10.1007/BF00326133

15. Geelhoed GW. Tracheomalacia from compressing goiter: management after thyroidectomy. Surgery. 1988;104(6):1100-8. PMID: 3194836 